\title{
Evaluación de la inteligibilidad del habla en español*
}

The evaluation of the intelligibility of speech in Spanish

\author{
Jorge Sommerhoff $\square^{1}$, Claudia Rosas ${ }^{2}$ \\ ${ }^{1}$ Universidad Austral de Chile, Instituto de Acústica, \\ Facultad de Ciencias de la Ingeniería, Valdivia, Chile. \\ e-mail: jsommerh@uach.cl \\ ${ }^{2}$ Universidad Austral de Chile, Instituto de Lingüística y Literatura, \\ Facultad de Filosofía y Humanidades, Valdivia, Chile. \\ e-mail: claudiarosas@uach.cl
}

\begin{abstract}
En el inglés de Norteamérica existe un corpus de palabras fonéticamente balanceadas (PB) para la medición de la inteligibilidad del habla, estandarizado por ANSI (American National Standards Institute) y correlacionado con la medición del STI (Speech Transmition Index). En el español no existe aún un corpus de palabras estandarizado que permita la comparación de ambos tipos de procedimientos. A partir de la correlación establecida del inglés, en este trabajo se ha evaluado el comportamiento de dos listas ya existentes del español que miden la inteligibilidad. Como resultado se entrega la ecuación que relaciona el \% de inteligibilidad de cada lista en función del STI medido. Se concluye que los resultados de las mediciones de inteligibilidad de las listas utilizadas difieren y que no se correlacionan con los resultados del STI, como sucede con el corpus inglés normalizado por ANSI.
\end{abstract}

Palabras clave: inteligibilidad, habla, medición.

In the North American English language there is a phonetically balanced (PB) word corpus for measuring speech intelligibility which has been standardized by ANSI and is correlated with measurements of STI. In the Spanish language little has been done in the development of list of words that fulfills the same ANSI objectives. Starting from the correlation established for English, the behaviour of two lists of words that measures intelligibility is analyzed. As a result, the equation that relates the percentage of intelligibility of each list in relation to the measured STI is given. It is concluded that intelligibility measurements differ from the lists used, and that none of the Spanish corpus is correlated with the STI as it occurs with the English corpus normalized by ANSI.

Key words: intelligibility, speech, test.

* Resultados parciales del proyecto de investigación DID UACh S-2005-50, cuyo Investigador Responsable es Jorge Sommerhoff, y actualizado en el proyecto Fondecyt 1070210 (2007), Investigadora Responsable Claudia Rosas y coinvestigador Jorge Sommerhoff.

1. INTRODUCCIÓN

En el estudio de la comunicación oral conviene considerar las condiciones acústicas del recinto donde esta se realiza. Al respecto, la experiencia nos permite comprobar que muchos espacios cerrados están lejos de alcanzar condiciones acústicas apropiadas para la transmisión del lenguaje en forma oral, en perjuicio de la comprensión del mensaje. En efecto, las condiciones acústicas inadecuadas de un recinto pueden afectar la comunicación por razones relacionadas directamente con la alteración material de la emisión; por ejemplo: la interpretación incorrecta de una orden o instrucción derivada de la confusión de los sonidos; o bien, indirectamente, mediante los efectos psicofísicos de fatiga, irritabilidad y desconcentración que afectan a los sujetos expuestos a un ambiente ruidoso (Kryter 1985).

El grado de inteligibilidad del habla ${ }^{1}$ depende básicamente de factores semánticos y físicos. Estos últimos, que ahora nos ocupan, están relacionados con el ambiente acústico donde ocurre la interacción (reverberación y ruido de fondo, principalmente) y los efectos que sus características producen en los sonidos que integran el texto (enmascaramiento del sonido de la voz producido por el ruido de fondo, por

\footnotetext{
1 Se ha utilizado "inteligibilidad del habla" en el sentido más tradicional y amplio que esta formulación tiene en el estudio de la voz: capacidad de comprensión del material lingüístico (Cf. Llisterri 1991: 118 y 119).
} 
ejemplo); como, también, con las características acústicas inherentes de los sonidos mismos (cantidad de energía acústica que comportan las vocales y consonantes). En este sentido, llama la atención que, aunque se reconoce que la inteligibilidad del habla está estrechamente relacionada con la percepción de las características asociadas a las consonantes: menor energía acústica -traducida en menor duración y predominio de frecuencias altas- (Carrión1998), este hecho no haya sido tradicionalmente considerado en la elaboración de pruebas de articulación ${ }^{2}$ del español que utilizan logatomos ${ }^{3}$; donde, por el contrario, se observa predominio de sonidos vocálicos.

El aspecto del material del sonido que tiene relación con los efectos producidos en la información concita el interés desde una perspectiva interdisciplinar fonética y acústica, y este será el enfoque utilizado en el presente trabajo ${ }^{4}$.

Para determinar el grado de inteligibilidad del habla se utilizan dos tipos de procedimientos de medición: subjetivos y objetivos.

Los procedimientos subjetivos se caracterizan por emplear seres humanos para medir la inteligibilidad. En Norteamérica este tipo de medición está normado por la ANSI (1989). Se trata de un proceso de medición estadístico que utiliza locutores entrenados que dictan una lista estandarizada de palabras en el sistema de comunicación (recinto, o recinto + sistema electroacústico) a oyentes especializados que las transcriben. La capacidad de los oyentes para identificar las palabras -traducida en aciertos- reflejaría la calidad de la transmisión, es decir, la inteligibilidad. Este tipo de mediciones tiene variadas aplicaciones conectadas con ámbitos sociales también diversos (educación, esparcimiento, ingeniería...). Normalmente, se utiliza para: evaluar la acústica natural de una sala de conferencia o de clases, evaluar un sistema de intercomunicación a distancia, o evaluar la contribución que un sistema electroacústico hace a la mejora de la inteligibilidad del habla en una sala.

Para realizar las pruebas de articulación exigidas por este tipo de procedimiento existen varias listas de palabras especializadas que son de uso común en Norteamérica. Por ejemplo, la norma de ANSI especifica la Prueba de la Rima Modificada (PRM), la Prueba de Diagnóstico de Rima (PDR) y un conjunto de veinte Listas de Palabras Fonéticamente Balanceadas (PB (phonetic balanced, en inglés)). Otros ejemplos de listas de palabras son: las pruebas de Aliteración de Diagnóstico, Diagnóstico Medial de la Consonante y Alfabeto de Ortografía.

Siendo el sistema biológico del ser humano, con todas sus características, el que finalmente puede juzgar la inteligibilidad de un sistema o sala, las pruebas estadísticas que usan locutores y oyentes especializados constituyen el método más exacto y fiable para su medición. Sin embargo, este método tiene una desventaja práctica; ya que debe contar con una lista adecuada de palabras y con personas preparadas para su locución y audición, hechos que dificultan su implementación.

Los procedimientos objetivos, por su parte, han surgido como respuesta a la necesidad planteada en el párrafo anterior de proporcionar métodos de medición comparativamente más eficientes; en cuanto a la cantidad de recursos humanos utilizados, rapidez en la aplicación del procedimiento y fiabilidad de los resultados obtenidos. Con este fin, se ha trabajado desde hace años en la búsqueda de una medición con máquinas que, sin menoscabo de la validez de los resultados sobre el aspecto de la inteligibilidad del habla que interesa averiguar, sea rápida y fácil de utilizar. Varios métodos han surgido en este esfuerzo, cuyos resultados se pueden clasificar en dos categorías básicas: (a) mediciones del campo reverberante, que agrupan las pruebas que informan sobre: las cualidades reverberantes del recinto y su relación con la inteligibilidad (\%Alcons (pérdida de definición de las consonantes)), la relación del campo directo al campo reverberante (por ejemplo, C50 - Speech Clarity), la relación entre sonido útil y sonido perjudicial (U50 y U80 (Useful-to-Detrimental Sound Ratios)), y la proporción entre la energía sonora temprana y tardía (Early-to-Late Sound Energy Ratio), por ejemplo; y (b) mediciones basadas en la relación señalruido, surgidas de la relación combinada entre el advenimiento de sistemas de comunicación electrónicos y la necesidad de medir la inteligibilidad del habla transmitida a través de ellos. Las más conocidas son el Indice de Transmisión del Habla (STI (Speech Transmition Index) $)^{5}$ resultado de los estudios

\footnotetext{
${ }^{2}$ El uso extendido del término "prueba de articulación” permite aludir inequívocamente a los métodos subjetivos que utilizan en sus mediciones de inteligibilidad seres humanos que transcriben lo que oyen o lo que alguien "articula" frente a los denominados métodos objetivos que utilizan máquinas (Cf. Gavilán 1988: 244).

3 Palabras sin sentido, pero que formalmente se ajustan al sistema de una lengua cualquiera (por ejemplo: charchar, criso que son palabras inventadas en español) (Cf. Llisterri 1991: 71).

4 Un ejemplo de este enfoque referido a las características acústicas de la reverberación y su incidencia en el sonido directo puede leerse en Rosas y Sommerhoff (2006: 235-249).

${ }^{5}$ El desarrollo inicial de este método, y debido al incipiente avance de la electrónica digital de entonces, obligó inicialmente al uso de un método más inexacto llamado RASTI (Rapid STI), el cual no analizaba todas las frecuencias comprometidas (Cf. Steeneken,
} 
realizados por Houtgast y Steeneken $(1973,1985 a$ y 1985b) y el Indice de Inteligibilidad del Habla (SII (Speech Intelligibility Index)). Estos autores determinaron que el comportamiento de la inteligibilidad del habla en una sala podía ser cuantificada por la Función de Transferencia de Modulación ${ }^{6}$ (MTF); es decir, la relación entre la magnitud de las fluctuaciones de la modulación de la señal original modulada (definidas por la envolvente de la intensidad de la señal) y el grado de preservación se preservan hasta llegar al auditor. Si bien, ambos sistemas pueden en determinadas ocasiones arrojar resultados no deseados, son considerados en la actualidad por los especialistas como los métodos de medición con máquinas más robustos, que utilizados bajo las condiciones correctas, muestran una buena correlación con las pruebas estadísticas ${ }^{7}$. El STI incluye en la función de transferencia de modulación los efectos de la reverberación, ruido y distorsión, características que lo convierten en un sistema muy potente.

En particular el STI tiene además otra ventaja: sus resultados han sido correlacionados con los listados de palabras normados por ANSI; hecho metodológicamente útil para el estudio de logatomos de cualquier idioma con fines comparativos e intereses prácticos. Con respecto a su funcionamiento, el STI utiliza una señal obtenida por síntesis que tiene las características de la señal de la voz humana. Lo que este instrumento hace es medir y comparar la profundidad de la modulación en el punto de recepción del recinto y la entregada a la fuente sonora en cada una de las varias bandas de frecuencia. El grado de reducción en la profundidad de la modulación en el punto de recepción está directamente relacionado con la pérdida de inteligibilidad cuyos resultados se distribuyen en una escala que va desde 0 (ininteligibilidad absoluta) a 1 (inteligibilidad absoluta).

La figura 1 muestra la relación entre el porcentaje de inteligibilidad medido subjetivamente, obtenida mediante la aplicación de una prueba de articulación de un corpus de palabras del inglés (logatomos CVC) y el porcentaje de medición objetiva que utiliza el STI (Houtgast y Steeneken 1985: 3-29).

En el idioma español se han encontrado dos listas de palabras que se utilizan para medir la inteligibilidad del habla. Sin embargo, no hay referencias sobre sus características lingüísticas: similitudes y diferencias. Tampoco hay -como sería deseable para efectos metodológicos y prácticos- referencias a un estudio sobre sus resultados en comparación con los del STI, y/o con los del corpus del inglés estandarizado por ANSI.

De lo anterior se desprende la necesidad de desarrollar corpus de palabras en el idioma español, que arrojen resultados semejantes en función del STI a las listas del idioma inglés establecidas en ANSI, con el fin de realizar investigaciones con resultados comparativos, independientemente que éstos utilicen individuos de habla española o individuos de habla inglesa.

La hipótesis de la investigación establece que diferentes listados de palabras elaborados para el idioma español con las combinaciones más recurrentes de letras pueden entregar resultados estadísticamente diferentes en las mediciones de articulación.

Figura 1

Relación entre prueba de articulación de un corpus de palabras del inglés y STI

\footnotetext{
J.M. y Houtgast 1985: 13-30).

${ }^{6}$ Señal original ; Señal transmitida . La variable m incluye los efectos del Tiempo de Reverberación y Relación Señal/Ruido.

${ }^{7}$ Se ha comprobado que bajo ciertas condiciones el STI también puede entregar resultados engañosos. En efecto, las reflexiones tardías y los ecos pueden alterar significativamente la medida. Pero puede dar medidas de inteligibilidad artificialmente bajas si se utilizan sistemas de compresión o limitación en el sistema electroacústico que reproduce la señal. Por otra parte, como el procedimiento de banda crítica ignora las frecuencias situadas bajo los $100 \mathrm{~Hz}$, puede dejar fuera frecuencias dentro de esos rangos que significativamente enmascaran la señal de la fuente.
} 
El objetivo de esta investigación es medir el porcentaje de articulación en distintas condiciones acústicas utilizando dos listados de palabras españolas ya publicados y comparar los resultados entre sí y con las respectivas mediciones de STI.

\section{MetodologíA}

2.1. El corpus. El corpus utilizado para la comparación de la eficacia en la medición de la inteligibilidad en función del STI corresponde a dos listas de logatomos españoles en uso: una publicada por Fuchs y Osuna (1965) y la otra, por Miñana (1969), tal como se muestra en las Tablas 1 y 2, respectivamente.

a) Características fonéticas de las listas. De la observación fonética se desprende que la suma de los monosílabos empleados en la lista de Fuchs y Osuna (en adelante, solo Fuchs) evidencia una proporción mayoritaria de la estructura consonante + vocal CV $(55 \%)$ coincidiendo, en este sentido, con la proporción mayoritaria constatada para el español Quilis (1999). Le sigue la estructura consonante + vocal + consonante CVC (20\%). Un tercer grupo, que representa un $25 \%$, lo integran estructuras diversas: vocal + consonante VC $(11 \%)$; consonante + vocal + consonante CVC $(8 \%)$; consonante + semiconsonante + vocal C SC V (5\%); y consonante + vocal + semivocal C V SV (1\%).

Por su parte, la lista de Miñana se caracteriza por una mayor proporción del tipo consonante + vocal + consonante CVC (44\%), alejándose parcialmente de la tendencia observada para el español. Le sigue la estructura consonante + vocal CV (19\%). Un tercer grupo, numeroso (37\%), pero variado, lo integran los siguientes tipos: consonante + vocal + semivocal C V SV $(16 \%)$; consonante + semiconsonante + vocal + consonante C SC V C (6\%); consonante + semiconsonante C SC (5\%); vocal + consonante VC (5\%); vocal + semivocal + consonante V SV C (2\%); vocal + semivocal V SV (2\%); y vocal + semivocal + consonante V SV C (1\%).

En cuanto a la realización articulatoria de los grafemas, aunque no se precisa información al respecto en ninguno de los corpus manejados, por una parte, la presencia en ambas listas de las grafías $S$ y $C$ (además de $z$ en Miñana) y de las grafías $b$ y $v$, en contextos similares sugieren la distinción articulatoria entre fricativas alveolares y dentales, y entre bilabiales y labiodentales, respectivamente (véase: ci vs. si; sap vs. zat; bar vs va, por ejemplo). Por otra parte, el uso de la grafía $g$ en un contexto idéntico al de $j$ en Fuchs (véase: je vs. ge), o su uso en get, contexto donde podría emplearse una $j$ que ya aparece en la palabra jol, en lista de Miñana, sugiere la articulación velar sonora [g].

Tabla 1

Lista publicada por Fuchs

\begin{tabular}{|c|c|c|c|c|c|c|c|c|c|}
\hline mar & rra & os & si & in & can & ar & bi & cie & cha \\
\hline sin & fa & bar & can & va & di & ben & pro & dun & gra \\
\hline to & pos & me & ña & cia & ya & su & na & sal & pe \\
\hline mu & ci & glu & se & ta & lo & ño & ge & rri & tu \\
\hline
\end{tabular}




\begin{tabular}{|c|c|c|c|c|c|c|c|c|c|}
\hline che & cra & co & do & tor & mi & te & sa & los & es \\
\hline es & du & un & al & doi & fui & le & dan & con & an \\
\hline que & pan & je & so & yo & to & dri & qui & la & pre \\
\hline no & de & les & ble & an & en & co & an & rre & rra \\
\hline per & ni & da & pe & dre & mas & mie & so & ce & bue \\
\hline te & mel & rre & ga & li & que & pal & ja & dos & me \\
\hline
\end{tabular}

Tabla 2

Lista publicada por Miñana

\begin{tabular}{|c|c|c|c|c|c|c|c|c|c|}
\hline res & va & la & quia & cen & sel & soir & cat & car & ted \\
\hline die & dot & de & pec & be & sar & od & ce & net & sai \\
\hline sap & co & sol & boc & nou & mou & ma & pac & at & al \\
\hline ta & map & quo & ya & lor & diar & zat & yot & con & roi \\
\hline cop & jol & me & leu & seir & ba & ven & dai & sia & nie \\
\hline dior & to & cies & nos & no & pol & nal & sen & que & ep \\
\hline fem & pe & loi & ras & fam & cat & tau & eir & es & les \\
\hline rei & ser & nai & quei & get & teu & rios & cam & dep & eu \\
\hline son & dog & rel & ram & au & la & nas & lied & rias & ye \\
\hline lau & dac & ga & pa & tai & sau & der & seu & tel & ren \\
\hline
\end{tabular}

2.2. Materiales y métodos. Previamente a la aplicación de la prueba de articulación fue necesario realizar un par de tareas: normalizar el dictado de logatomos y mantener las condiciones de emisión acústica lo más semejante posible con el objeto de permitir comparaciones válidas.

a) Grabación de las listas. Todos los sonidos fueron articulados según la norma fonética que rige la interpretación para el español hispanoamericano ${ }^{8}$. Así, las grafías $v$ y $b$ se articularon como bilabial [b]; $s, z$ y $c+e, i$ se articularon como alveolares [s]; $j$ y $g+e, i$ se articularon como velares sordas [x]. Las grabaciones de las listas se realizaron en una sala anecoica y con el micrófono en campo cercano con el fin de no agregarle campo reverberante a la señal. Las señales fueron procesadas de tal manera de dejar todos los logatomos separados a una distancia de 1,5 s mediante frases introductorias del tipo "pongan atención", "ahora escuchen", "escuchen a continuación", entre otras. Se realizaron diferentes dictados haciendo variar el orden de los logatomos con el fin de evitar la memorización de los oyentes.

\footnotetext{
${ }^{8}$ Cf. Martínez Celdrán (1996:42-47).
} 
Finalmente, se normalizó digitalmente la potencia acústica de las grabaciones emitidas, ajustándola a la de la voz de una persona normal.

El paso siguiente fue la aplicación de la prueba de articulación y su correspondiente medición. Para ello hubo que definir los informantes y los recintos de aplicación.

a) Informantes. Correspondieron a estudiantes universitarios, de ambos sexos, auditivamente sanos y elegidos al azar, los que se dividieron en 6 grupos de 5 individuos cada uno.

b) Recintos. Se utilizó dos tipos de salas acústicamente diferenciadas: (a) un tipo para obtener valores de STI menores a 0,4 (acústicamente deficientes); y otro, para conseguir valores de STI entre 0,4 y 0,8 (acústicamente aceptables). Como la variabilidad perseguida de STI en la misma sala se obtiene mediante distintas distancias del auditor con respecto a la fuente de sonido los estudiantes elegidos fueron distribuidos, rotatoriamente, en puntos estratégicos de la sala desde donde escribieron, en plantillas diseñadas para el efecto, las palabras escuchadas. Las salas, en cuestión, utilizadas corresponden a la Sala $6308^{9}$ del Edificio 6000 (Campus Miraflores) y a la Sala Auditorio (Campus Teja), Universidad Austral de Chile ${ }^{10}$.

c) Mediciones. Para medir el STI se utilizó el medidor NTI Acoustilyzer junto con la fuente sonora NTI Talk Box. Esta fuente tiene como característica imitar la voz humana, tanto en su potencia acústica como en su directividad ${ }^{11}$. También dispone de una tarjeta Compact Flash, que permite el almacenamiento de las señales que emite, y que en este trabajo corresponden a la señal STI y a los dos corpus de logatomos. Las mediciones de STI están pareadas con las mediciones de inteligibilidad.

En los mismos puntos donde fueron ubicados los auditores se midió el STI de acuerdo al montaje que se muestra en la figura 2 .

Figura 2

Montaje para las mediciones en sala de prueba

\section{RESULTADOS}

\footnotetext{
${ }^{9}$ Posteriormente demolida (febrero de 2007).

${ }^{10}$ La selección de estas salas obedece a la presencia hallada -durante datos derivados de un amplio rango de STI- tareas de medición previas, condición requerida para poder modelar las curvas obtenidas.

${ }^{11}$ La fuente sonora "Talk Box" reemplaza a la voz humana. Su potencia acústica y directividad son semejantes a las emitidas por la voz hablada de una persona en condiciones normales. Esta potencia viene calibrada de fábrica y no tiene, por tanto, posibilidades de ser alterada con algún control.
} 
La figura 3 muestra los puntos obtenidos correspondientes al \% de Inteligibilidad y STI de cada medición, como también, las curvas polinómicas calculadas con dichos puntos. La desviación estándar de los puntos con respecto a la curva polinómica es de 8,6\% para el caso de Miñana y 8,8\% para el caso de Fuchs. La Ecuación (1) y la Ecuación (2) muestran la función que relaciona el\% de Inteligibilidad en función del STI de las listas de Miñana y Fuchs, respectivamente.

$$
\begin{aligned}
& I A=-0,1944 \mathrm{STI}^{3}-2,1131 \mathrm{STI}^{2}+3,5918 \mathrm{STI}-0,4767 \\
& I A=2,3993 \mathrm{STI}^{3}+6,304 \mathrm{STI}^{2}+5,3057 \mathrm{STI}-0,4799
\end{aligned}
$$

Donde $I A=\%$ de Inteligibilidad .

3.1. Diferencias entre las curvas medidas. La figura 4 muestra las tres curvas polinómicas de los corpus de Miñana, Fuchs y Houtgast. El gráfico se ha dividido en rangos de categoría de calidad acústica con respecto a su inteligibilidad, de acuerdo a lo establecido por los estándares del STI.

En la Tabla 3 se han establecido las diferencias en el comportamiento de los corpus con respecto a los rangos de calificación de calidad. Se observa que el corpus de Miñana es más útil a la hora de evaluar el\% de Inteligibilidad que el de Fuchs, ya que tiene rangos mejor distribuidos en la escala de calificación. A pesar de ello, el corpus de Miñana está más alejado de la diagonal que el corpus utilizado en el idioma inglés para evaluar el\% de Inteligibilidad.

Tabla 3

Clasificación de calidad en función del\% de Inteligibilidad

\begin{tabular}{|l|c|c|c|c|c|}
\hline \multirow{2}{*}{ \% Inteligibilidad Listas } & \multicolumn{5}{|c|}{ Clasificación de Calidad } \\
\cline { 2 - 6 } & malo & deficiente & regular & bueno & excelente \\
\hline Miñana & $<40 \%$ & $40 \%$ a $69 \%$ & $69 \%$ a $88 \%$ & $88 \%$ a 95\% & $95 \%$ a $100 \%$ \\
\hline Fuchs & $<60 \%$ & $60 \%$ a $85 \%$ & $85 \%$ a $95 \%$ & $95 \%$ a $100 \%$ \\
\hline Houtgast & $<32 \%$ & $32 \%$ a $62 \%$ & $62 \%$ a $87 \%$ & $87 \%$ a $95 \%$ & $95 \%$ a $100 \%$ \\
\hline
\end{tabular}

\section{CONCLUSIONES}

La primera conclusión importante -y paso previo para la implementación de cualquier procedimiento articulatorio tendiente a la evaluación de la inteligibilidad del habla- es que en similares condiciones acústicas distintos corpus lingüísticos generan evaluaciones de \% de inteligibilidad diferentes.

En segundo lugar, los resultados de este estudio indican que, en efecto, los corpus en español comparados no se pueden correlacionar con el STI de igual forma que el corpus del inglés normalizado por ANSI. Al respecto se comprueba que las curvas polinómicas de los corpus españoles analizados muestran diferencias de hasta un 22\%; siendo éstas mayores en los rangos de STI calificados como "malo", "deficiente" y "regular", en tanto que en el rango "excelente" las curvas concuerdan, lo que es coincidente con la lógica esperada. Con todo, la lista de Miñana es más útil para medir \% de inteligibilidad en el idioma español; ya que el rango de resultados que ofrece está mejor distribuido en las categorías de calidad y es relativamente más próximo a lo que ofrece el corpus inglés normalizado por ANSI.

Por último, como producto, de las constataciones anteriores, se mantiene la necesidad de desarrollar corpus de palabras en el idioma español, que arrojen resultados semejantes en función del STI a las listas del idioma inglés establecidas en ANSI, con el fin de realizar investigaciones con resultados comparativos, independientemente de que éstos utilicen individuos de habla española o de habla inglesa. Este hecho plantea preguntas que abren la vía para trabajos posteriores de orden acústico y perceptivo. 
Para tal efecto, conviene señalar, de acuerdo a lo aquí evidenciado, que el diseño de una lista de logatomos para la medición de la inteligibilidad en español comparable con el STI -tema que este trabajo inicia- debe necesariamente considerar en su estructura silábica una proporción mayor de consonantes ${ }^{12}$.

OBRAS CITADAS

ANSI Standard S3.2-1989. 1989. Method for Measuring the Intelligibility of Speech Over Communication Systems. ANSI, New York.

Carrión Isbert, A. 1998. Diseño acústico de espacios arquitectónicos. Barcelona: Edicions UPC. 45.

Fuchs, G. L. y J. Osuna. 1965. "Medición de Inteligibilidad". Memoria de las Primeras Jornadas Latinoamericanas de Acústica. Universidad Nacional de Córdoba.

Gavilán, J. 1988. Fonoaudiología para educadores. Madrid: Universidad Nacional de Educación a Distancia.

Houtgast, T. y J. M. Steeneken. 1973. "The modulation transfer function in room acoustics as a predictor of speech intelligibility". Acustica, 28 (1): 66-73.

- 1985a. "The Modulation Transfer Function MDT in room acoustics". B \& K Tech. Rev., 3, 3-29.

- 1885b. "A review of the MTF concept in room acoustics and its use for estimating speech intelligibility in auditoria". J. Acoust. Soc. Am. 77: 1069-77.

Kryter, K. D. 1985. The effects of noise on man, Academia Press.

Llisterri, J. 1991. Introducción a la fonética: el método experimental. Barcelona: Editorial Anthropos.

Martínez Celdrán, E. 1996. El sonido en la comunicación humana. Barcelona: Ediciones Octaedro.

Miñana, P. 1969. Compendio práctico de acústica. Barcelona: Labor.

Quilis, A. 1999. Tratado de fonología y fonética españolas. Madrid: Gredos.

Rosas, C. y J. Sommerhoff. 2006. "Variabilidad idiofónica en español como herramienta forense". Estudios Filológicos 41: 235-249.

Steeneken, J.M. y T. Houtgast. 1985. "RASTI: a Tool for Evaluating Auditoria”. B \& K Tech. Rev. 3: 13-30.

\footnotetext{
12 En efecto, una prueba de articulación consonante + vocal + consonante cvc aplicada por un estudiante de ingeniería en acústica (tesista en proceso), Rodrigo Muñoz, permite obtener un rango de dispersión comparativamente mayor que el de las listas de Fuchs y Miñana, manejadas, hecho que reafirma esta hipótesis, si bien de manera parcial por la cantidad de experimentos que resta hacer. Conviene hacer notar que dicha prueba forma parte de las tareas de sondeos preliminares del Diseño general de elaboración de un listado de logatomos en español (en proceso), que considera alrededor de 1.000 logatomos y distintas variables relacionadas con la estructura silábica (tipo de segmento, posición y combinación).
} 